\title{
List of Notes
}

Filenames and paths -6

Installing community-contributed software -9

Log-Files and folder structures - 14

Listing cases $-\mathbf{1 8}$

Using the Stata documentation -23

Assigning VS equality testing - $\mathbf{3 1}$

New variables and labels in one step - 34

The Fre command - 46

Dealing with outliers $-\mathbf{5 4}$

Statistical significance $-\mathbf{7 2}$

What about ANOVAs? $\mathbf{8 9}$

Confidence intervals $-\mathbf{9 3}$

Nested models — 106

Macros 117 